\title{
Editorial
}

\section{Nanostructured Carbon Materials}

\author{
Myoung-Woon Moon, ${ }^{1}$ Ho-Young Kim, ${ }^{2}$ Aiying Wang, ${ }^{3}$ and Ashkan Vaziri ${ }^{4}$ \\ ${ }^{1}$ Korea Institute of Science and Technology (KIST), Seoul 136-791, Republic of Korea \\ ${ }^{2}$ Seoul National University, Seoul 151-744, Republic of Korea \\ ${ }^{3}$ Ningbo Institute of Materials Technology and Engineering (NIMTE), Chinese Academy of Sciences, Ningbo 315201, China \\ ${ }^{4}$ Northeastern University, Boston, MA 02155, USA
}

Correspondence should be addressed to Myoung-Woon Moon; mwmoon@kist.re.kr

Received 30 April 2015; Accepted 30 April 2015

Copyright (c) 2015 Myoung-Woon Moon et al. This is an open access article distributed under the Creative Commons Attribution License, which permits unrestricted use, distribution, and reproduction in any medium, provided the original work is properly cited.

Nanostructured carbon materials have attracted great attention due to their capability for use in various fields such as energy, environment, water, or biomedicine. Nanostructured carbon materials form various allotropes in zero-, one-, two-, and three-dimensional nanoscale such as fullerene, carbon nanotubes (CNTs), graphene or nanocarbon coating, and diamond or porous carbon, respectively. Furthermore, carbon materials can have new functions in electrical, physical, or chemical properties by assembling or architecturing with different functional nanomaterials, such as nanocomposites of CNTs with functional nanoparticles, carbon nanocoatings with functional metal element or graphene modified with carbon materials, and metal or oxide nanomaterials.

The major goals of this special issue are to find novel fabrication methods for nanostructured carbon materials and the assembling or architecturing of carbon nanomaterials with metal or oxide nanomaterials for novel functionalization in up-to-date applications. The issue includes research papers and article reviews covering a wide range of current progress on nanostructured carbon materials in the wide range of the topics from the fundamentals to the application with nanostructured carbon materials.

In this special issue, some articles are addressing the above-mentioned issues in the form of review. The contribution by S.-N. Lu et al. is providing a review for the state of the art on the applications of nanostructured carbon materials, including carbon nanotubes, carbon nanofibers, and graphene oxides. Its coverage ranges from the preparation process of the nanostructured materials to the applications such as mechanical reinforcement, self-sensing detectors, and self-heating element. One of the review articles by C. Luo et al. is dealing with the application and performance of carbon nanotubes in fuel cells. The authors provide a review from the noble metals as catalysts to the performance of fuel cell. Treated are the method used to reduce the platinum, the effect of carbon nanotubes on the fuel cell, improving the performance of fuel cell catalysts, the interaction between catalyst and carbon nanotube support, and the synthetic conditions of carbon nanotube supported catalyst.

A research article by M. L. García-Betancourt et al. is presented on the magnetic and electrical properties of nitrogen-doped multiwall carbon nanotubes fabricated by a modified chemical vapor deposition method. By the modification of the CVD configuration, the authors suggested a synthesis way for the morphology control for the nitrogen-doped nanotubes. They suggested that the semiconducting nature of the nitrogen-doped CNT can be shown with water and low $\mathrm{NaCl}$ during synthesis processing. Another research article by X. Zhang et al. shows that the multiwalled CNTs (MWCNTs) by $\mathrm{CF}_{4}$ plasma treatment for $\mathrm{SF}_{6}$ decomposition component detection are presented on the enhancement of gas-sensing characteristics. MWCNTs, modified by dielectric barrier discharge of $\mathrm{CF}_{4}$ plasma, show that the gas-sensing effect to $\mathrm{H}_{2} \mathrm{~S}$ and $\mathrm{SO}_{2}$ was effective for the treatment duration since modified MWCNTs contained a low number of impure particles on the surface as well as short length and grafted-F polar functional groups.

Synergetic effects of mechanical properties on graphene nanoplatelet and MWCNT hybrids reinforced epoxy/carbon fiber composites were explored by P.-N. Wang et al., who 
provide an outstanding synergetic effect on the graphene nanoplatelets (GNPs) and MWCNTs hybrids. They reported that reinforced epoxy composite and epoxy/carbon fiber composite laminates enhance their mechanical properties over that of neat laminates.

Two research articles present the functional nanocarbon materials of diamond films and biocarbon nanofibers. A research on $\mathrm{N}$-type conductive ultrananocrystalline diamond films grown by hot filament CVD is presented by M. Mertens et al. The research article contributed by X. Huang et al. explores the study of electromagnetic interference (EMI) shielding materials comprised of Ni-Co coated on weblike biocarbon nanofibers via electroless deposition. EMI shielding materials made of $\mathrm{Ni}$-Co were coated on web-like biocarbon nanofibers prepared by electroless plating. Biocarbon nanofibers (CF) were obtained using bacterial cellulose pyrolyzed at $1200^{\circ} \mathrm{C}$. The EMI shielding efficiencies of the composites were shown to be significantly higher than that of $\mathrm{CF}$ at the same mass fraction.

\title{
Acknowledgments
}

The guest editors would like to thank the authors for their contributions to this special issue and the reviewers for their time and dedication.

\author{
Myoung-Woon Moon \\ Ho-Young Kim \\ Aiying Wang \\ Ashkan Vaziri
}



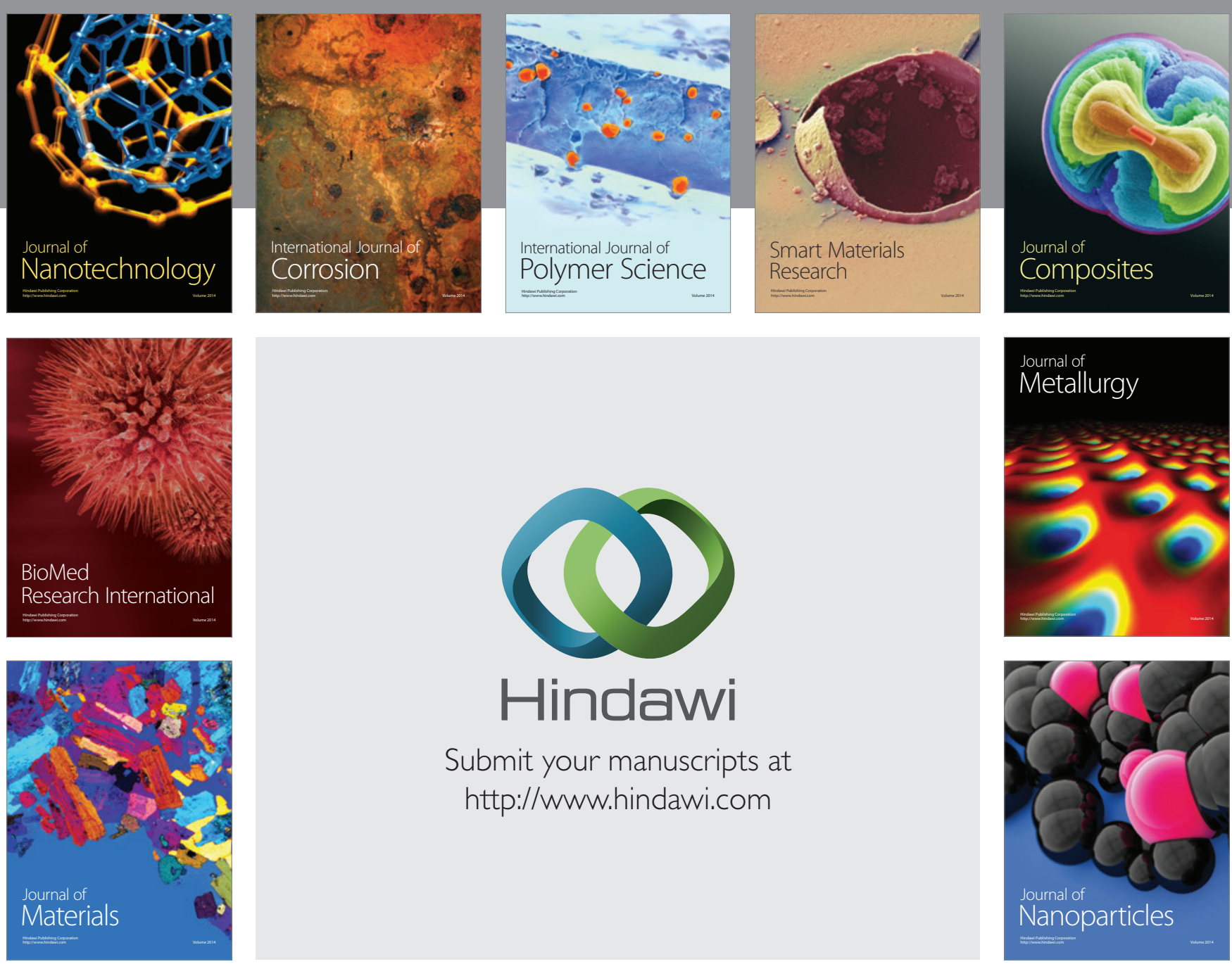

Submit your manuscripts at http://www.hindawi.com
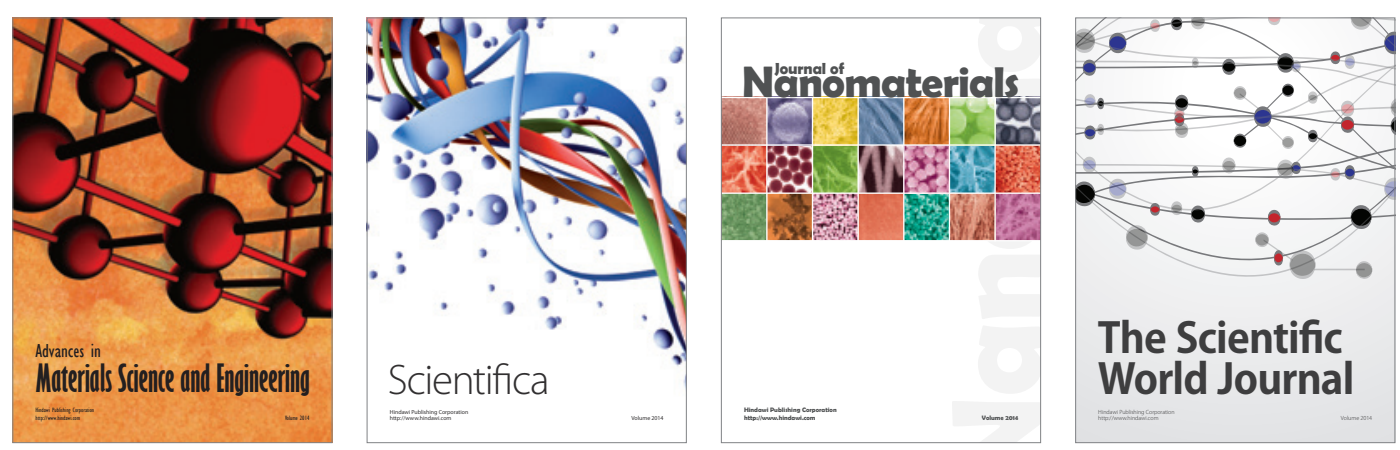

\section{The Scientific World Journal}
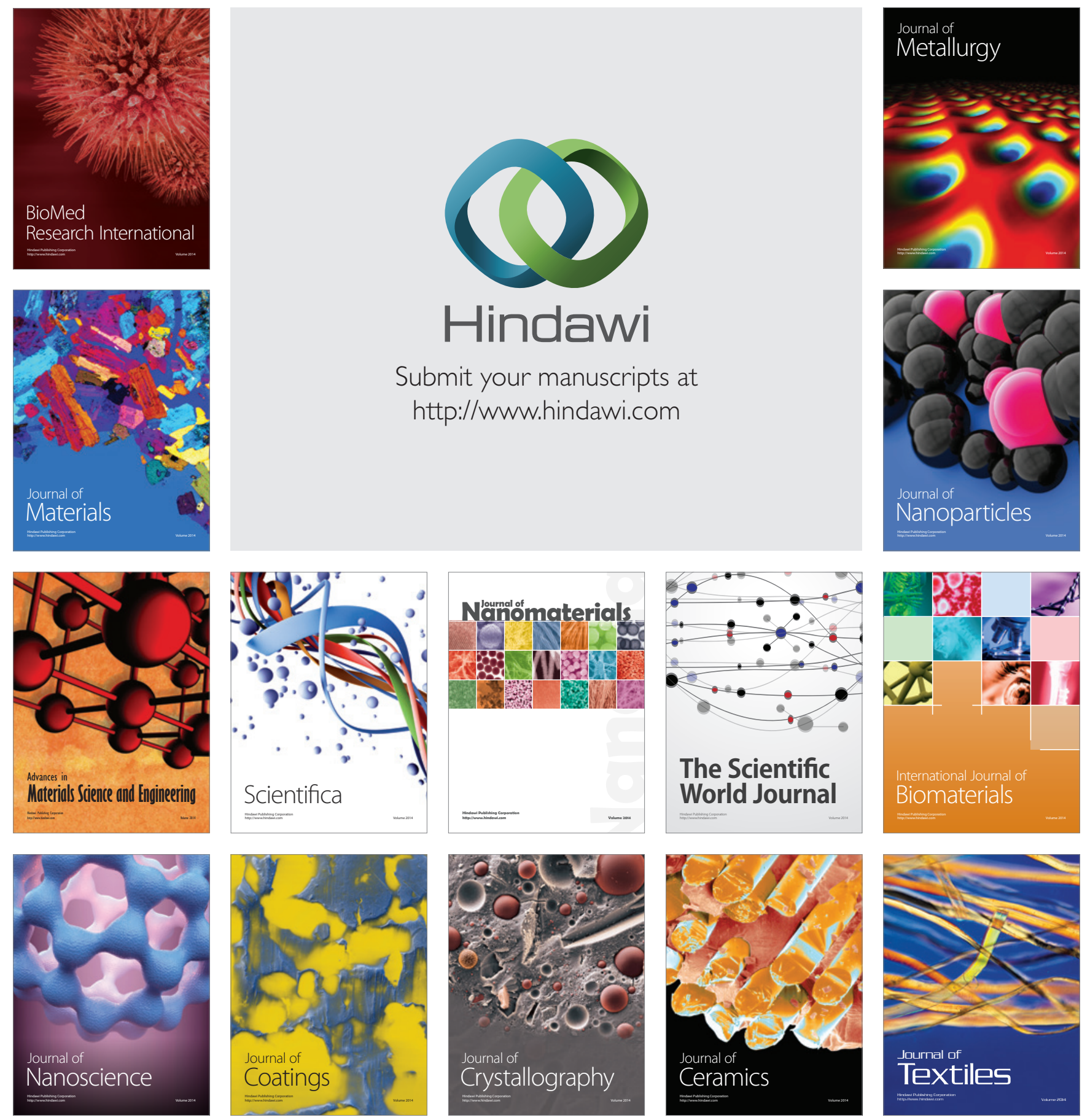